\title{
Anthelmintic resistance in goats in Peninsular Malaysia
}

\begin{abstract}
Ninety-six randomly selected farms, located throughout peninsular Malaysia, were surveyed for goat nematodes resistant to benzimidazoles (BZ). On 33 farms BZ resistance was demonstrated by means of an egg hatch assay. Haemonchus contortus was found to be the main species involved in anthelmintic resistance. There was a positive association between the frequency of anthelmintic treatments on a farm and the presence of benzimidazole resistance. To assess the value of the egg hatch assay, faecal egg count reduction (FECR)tests were also performed on 20 farms. On six farms the LD50 of thiabendazole (TBZ)was less than $0.10 / \sim \mathrm{g} \mathrm{ml}-1$ and the FECR higher than $95 \%$ and on ten farms with an LD50 TBZ of over $0.10 / \mathrm{tg}$ ml- $\sim$ a FECR of less than $95 \%$ was measured. On four farms the FECR was less than $95 \%$, although the egg hatch assay showed LD50 TBZ values of less than $0.10 \sim \mathrm{g}$ ml- $\mathrm{t}$ and on two of these three farms a controlled efficacy test confirmed the presence of BZ resistant $\mathrm{H}$. contortus. From these results it can be concluded that the egg hatch assay underestimated the true incidence of benzimidazole resistance. Levamisole resistance was detected with a FECR test on two of ten farms investigated.
\end{abstract}

Keyword: Haemonchus contortus, Goat, Resistance-Nematoda, Benzimidazoles 